\title{
EcoLocate: A heterogeneous wireless network system for wildlife tracking
}

\author{
Andrew C. Markham, Andrew J. Wilkinson \\ Department of Electrical Engineering \\ University of Cape Town \\ Cape Town, South Africa \\ mrkand007@uct.ac.za
}

\begin{abstract}
All research to date using wireless networks for wildlife tracking has concentrated on monitoring a single species, using large GPS enabled collars. These collars are too heavy to attach on smaller animals. Rather than omit small animals from the tracking spectrum, we show how a fusion of GPS tracking (where applicable) and an improved version of VHF tracking can result in a system which is able to track a wide range of animal species using the same underlying wireless network for information transfer. Tags are equipped with radio transceivers, which are used to both transmit and receive beacons. Received beacons are used to construct radio proximity maps which characterize co-location of various animals at different points in time. Furthermore, as the locations of some nodes are known, coarse estimates of animal locations can be determined, especially around focal points such as waterholes. We present the components of our system and discuss our prototype implementation.
\end{abstract}

Keywords-Wildlife tracking; GPS; VHF; Wireless Network; beacon; proximity detection

\section{INTRODUCTION}

No research to date has considered the incredible diversity in the Animal Kingdom when designing a wireless network for wildlife monitoring and tracking. The preeminent and frequently cited example of wildlife wireless sensor networks is ZebraNet [1], [2]. As the name of the project suggests, zebras were equipped with solar powered GPS tracking collars which transferred information by using bi-directional wireless links to form a network. Information was transferred using a simple flooding protocol such that all collars would share the same data. The motivation for ZebraNet is that by just encountering one or two animals, data from all the other animals can be retrieved, greatly reducing the logistics involved in locating a large number of animals in order to perform a complete download. Whilst their pioneering work provided some valuable lessons, especially regarding the difficulties involved in real world deployment, they only considered one single species of animal. Although there is nothing preventing the same ZebraNet collars from being placed on similar sized or larger animals (such as buffalo or elephant), smaller mammals cannot be equipped with such heavy and large collars. This is due to guidelines from the wildlife tracking community, who recommend that tag weight should not exceed $3-5 \%$ of animal bodyweight [3]. Thus, a valuable cross-section of the Animal Kingdom is ignored or omitted, whereas zoological researchers need to be able to monitor as wide a range as possible. From multi-species data, they can infer both inter- and intra- specific behaviour, and propose guidelines for wildlife management. However, the power consumption of current GPS receivers precludes their use on smaller animals. Although the receiver itself is light (in the order of ten grams) the battery capacity required to keep the unit operating for a significant monitoring duration (a year or more) is large (a few hundred grams).

However, this does not mean that smaller wildlife should be omitted entirely from the spectrum of wildlife tracking. Merely because GPS technology cannot be used, this does not mean that no technology at all should be used. Rather, we turn to the original technology that was placed on wildlife before the advent of satellite location, namely VHF tracking and triangulation.

This paper first reviews conventional VHF tracking illustrating some of the difficulties of this technique. Next, we discuss how the concepts behind VHF tracking can be improved by equipping tags with bi-directional radiotransceivers. The components of our system are then introduced and power consumption and lifetime are discussed. Finally, we compare similar work, pose future directions and draw conclusions.

\section{CONVENTIONAL VHF TRACKING}

VHF tracking involves placing small RF transmitters on animals [4]. These emit a signal every few seconds on a dedicated narrow frequency band. Researchers then estimate the location of an animal by obtaining bearings to the animal from two or more known locations using directional Yagi antenna and a handheld receiver. The strength of the signal is typically gauged manually using headphones, and the bearing is obtained by rotating the antenna in the direction of maximal loudness. Obtaining location estimates is a laborious and time consuming process, especially if large numbers of animals are required to be located [3], [4]. Furthermore, location estimates have accuracies in the range of tens to hundreds of metres as opposed to the impressive $(<10$ [m] CEP [5]) accuracy of GPS receivers. Even though the estimates are much less accurate, the simplicity and small size (a few grams) of VHF beacons 
make them an attractive (and in most cases, the only) option for smaller animals.

The goal of our research is to provide a framework in which VHF tracking and GPS receivers are combined with the increased functionality provided by the wireless network. Large animals are equipped with full function GPS tracking collars with radio transceivers for two way communication, whilst the smaller animals are just equipped with the lightweight radio transceivers. Operation is controlled by a microcontroller and collars are powered by a battery (augmented in some cases with a solar panel). In the contemporary VHF scenario, the collars have a transmit-only radio unit, but we show how beacons can be used to obtain coarse positional or contextual information through receiving other beacons within radio range.

\section{BEACONS FOR PROXIMITY DETECTION}

Each $\operatorname{tag}^{1}$ in the network periodically emits a beacon signal. Where this differs from conventional VHF tracking is that the beacon signal is a digital packet as opposed to a single pulse. Amongst other data, the transmitted packet of data contains the unique ID (identification) code of the tag. Another difference with existing VHF technology is that all the tags transmit on the same channel. Nodes access the channel randomly, which obviates difficulties inherent in time synchronizing a sparse, mobile network, but also results in periodic packet loss through collision, when two or more tags attempt to access the medium simultaneously. However, the average time interval between beacon transmissions is long (typically $3 \mathrm{~s}$ ) compared to the time required to transmit a packet $(6 \mathrm{~ms})$. Furthermore, as the network is extremely sparse, the number of nodes in close radio proximity is typically very small resulting in a low probability of collision.

In standard VHF tracking, there are many transmitting beacons and a single receiver. However, as tags in our system are equipped with a bi-directional radio transceiver, tags can both transmit and receive beacons. Thus, a tag periodically listens to the beacon channel for a length of time and captures the ID numbers of the nodes within radio proximity. As all the beacons transmit on the same channel, a tag does not have to scan through multiple frequencies, reducing the overall scan time. Nodes store the identities of the beacons that were overheard within the listening window, along with a timestamp indicating time of reception. Typical activity on the beacon channel is illustrated in Fig. 1. This data is then transferred through the multi-hop wireless network to ultimately reach a base-station, where a logical connectivity map can be constructed. The connectivity map is a timevarying representation of the radio proximity of various tags. Based on this data, inferences can be made about the habits and behaviours of animals.

This approach dramatically increases the usefulness of the tracking system, as the map essentially characterizes the colocation of tags, albeit coarsely. This information can be used by researchers to determine if a certain animal is frequently in contact with other particular animals and also captures the time

\footnotetext{
${ }^{1}$ The terms tag, collar and node are used interchangeably in this paper
}

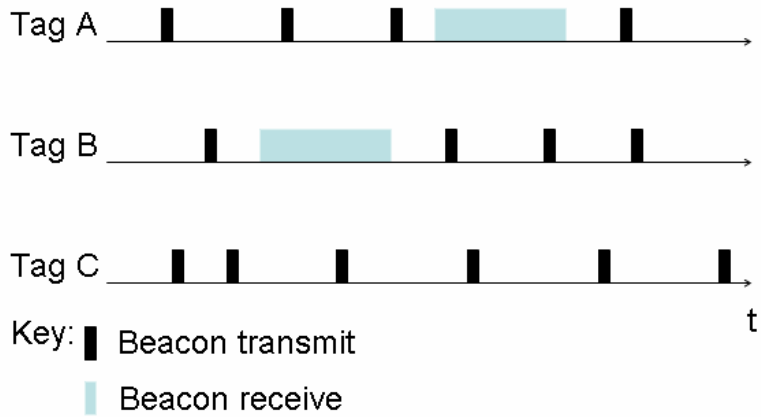

Figure 1. Timeline showing tag activity in the beacon channel. When tags are not transmitting nor receiving, they enter a low power sleep mode to conserve power.

dependency of this relationship, which can provide valuable insight into animal behaviour. For example, some animals, such as leopards, are solitary except during the mating season. By tagging these creatures, and monitoring their proximity, the genetic lineage can be discovered which provides invaluable information to the field of wildlife research [6]. Furthermore, the proximity of animals to human settlements or park boundaries can be monitored which can provide an early warning about rogue or problem animals.

However, this is not all that the time varying connectivity map can tell. If the locations of some nodes are known (either in fixed locations or mobile and equipped with GPS receivers), the approximate location of an animal not carrying a GPS receiver can be determined. With an increasing number of simultaneous location estimates, the accuracy of the predicted position can be refined. Such location estimates, whilst very coarse in relation to GPS fixes, can provide important information about the times at which animals visit certain locations such as waterholes and how long they remain in that area. Thus, this moves from simple (and laborious) VHF tracking to a system which can generate a wealth of interesting and useful data. The ramifications of such an approach are not just directed towards the field of wildlife research, but also to more general aspects of wildlife management. Thus decisions about the number and placement of waterholes, size and range of game reserves, and allocation of resources can be performed in a scientific and informed manner.

The system components which are used to acquire and transfer this data are now introduced.

\section{SYSTEM COMPONENTS}

As mentioned previously, the highly diverse Animal Kingdom demands a widely heterogeneous solution. To this end, the wireless network is comprised of four different classes of tags, with increasing levels of functionality. In addition to increased features, the higher level nodes have the same functionality of all the lower level nodes. This leads to a unified design approach, as they all run the same basic firmware except certain functions are disabled based on their class. The development cycle is consequently simplified as only one source code has to be maintained - the class of the tag is chosen either at design time (through conditional compiler switches) or dynamically at run time in the field (through 
assessment of their relative rank in the system [7]). The tags in increasing order of functionality (and correspondingly increasing order of power consumption and weight) have been termed Marker nodes, Spotter nodes, Pack nodes and Basestation nodes.

In our prototype implementation we have used the Microchip PIC18LF series of microcontrollers [8] and the Nordic NRF905 UHF radio transceiver [9] operating in the 868 $\mathrm{MHz}$ ISM license free (European and African) band. Tag operation is controlled using a finite state machine based operating system, where the choice of available states is controlled by the class of the tag. The radio transceiver achieves a quoted transmission range of $300 \mathrm{~m}$ at $+10 \mathrm{dBm}$ output power [9], but tests in the field show that the range is more typically 150 to $200 \mathrm{~m}$. Depending on local topographic features, the radio transmission range can drop to as low as 30 $\mathrm{m}$. For GPS enabled nodes, the $\mathrm{u}$-blox LEA-4P receiver is used to determine location [10]. Information is stored either in the FLASH memory of the microcontroller itself, or using an SD card which provides an inexpensive method of storing large (512 $\mathrm{Mb}$ or more) volumes of data. Some nodes have also been equipped with solar panels to provide a renewable source of energy. Figures regarding power consumption are thus based on these set of components, assuming a nominal $3 \mathrm{~V}$ power source. Typical values for current consumption, as well as predicted lifetime based on battery with $1 \mathrm{Ah}$ of capacity are shown in Table 1.

\section{A. Marker Tags}

Marker tags are the simplest type of tag in the network and have extremely low power consumption. Thus, this means that they can be fitted with a lightweight battery, making them suitable for tagging small animals. These tags just act as beacons, periodically emitting ('marking') their ID and other salient information, such as temperature and movement parameters in a packet. Marker tags never listen for other node's IDs and consequently consume a miniscule amount of power as they spend the majority of their time in low power sleep mode. Tags randomly wake up to transmit a packet before returning to sleep mode. Using the prototype node, if a node transmits its ID on average every three seconds, it will last for approximately 380 days per Ah of battery capacity. With two small coin cell batteries (such as CR-2032 [11]), a year of life can be obtained from a tag with total weight of 15 g.

Two unpackaged Marker tags are shown in Fig. 2, demonstrating their small size. In this picture, the antenna has not yet been attached. The 28 pin 18LF2620 microcontroller is used for the Marker tag and is wired up to the transceiver board. Although labour intensive, this results in a lower overall tag weight.

\section{B. Spotter Tags}

These tags provide all the functionality of the Marker nodes but periodically 'spot' or listen to other nodes within their radio range. They store the overheard node IDs and any other transmitted data in memory, along with a timestamp. When in range of a pack or base-station tag they transfer the stored information through wireless network. However, they only act
TABLE I. POWER CONSUMPTION OF THE VARIOUS CLASSES OF TAGS WITHIN THE ECOLOCATE SYSTEM

\begin{tabular}{|c|c|c|c|c|c|}
\hline Tag Type & Operation & Duty cycle & \begin{tabular}{|l|} 
Current \\
$(\mathrm{mA})$
\end{tabular} & $\begin{array}{l}\text { Mean Current } \\
(\mathrm{mA})\end{array}$ & $\begin{array}{l}\text { Lifetime per } \\
\text { Ah (days) }\end{array}$ \\
\hline \multirow{2}{*}{ Marker } & Sleep & $99.8 \%$ & 0.04 & \multirow{2}{*}{0.11} & \multirow{2}{*}{386} \\
\hline & \begin{tabular}{|l} 
Beacon Tx \\
\end{tabular} & $0.2 \%$ & 34 & & \\
\hline \multirow{4}{*}{ Spotter } & Sleep & $99.2 \%$ & 0.04 & \multirow{4}{*}{0.21} & \multirow{4}{*}{200} \\
\hline & Beacon Tx & $0.2 \%$ & 34 & & \\
\hline & Beacon Rx & $0.5 \%$ & 15 & & \\
\hline & Upload & $0.1 \%$ & 26 & & \\
\hline \multirow{4}{*}{ Pack } & Sleep & $98.3 \%$ & 0.04 & \multirow{4}{*}{0.44} & \multirow{4}{*}{94} \\
\hline & Beacon Tx & $0.2 \%$ & 34 & & \\
\hline & Beacon Rx & $0.5 \%$ & 15 & & \\
\hline & \begin{tabular}{|l} 
Route \\
\end{tabular} & $1.0 \%$ & 26 & & \\
\hline \multirow{5}{*}{$\begin{array}{c}\text { GPS } \\
\text { Enabled } \\
\text { Pack }\end{array}$} & Sleep & $95.0 \%$ & 0.04 & \multirow{5}{*}{1.71} & \multirow{5}{*}{24} \\
\hline & \begin{tabular}{|l} 
Beacon Tx \\
\end{tabular} & $0.2 \%$ & 34 & & \\
\hline & Beacon Rx & $0.5 \%$ & 15 & & \\
\hline & Route & $1.0 \%$ & 26 & & \\
\hline & GPS fix & $3.3 \%$ & 38 & & \\
\hline
\end{tabular}

as leaf or end nodes in the network and do not route other nodes' packets. Their power consumption is higher than the simpler Marker type tags, as they have to remain awake in active receive mode for twice the average inter-beacon time due to the unsynchronized nature of the channel access. Thus the receive window is six seconds long if the average interbeacon transmission time is three seconds. For a Spotter tag which transmits a beacon every three seconds and listens for beacons every ten minutes, it will survive for approximately 280 days per Ah of battery capacity. Thus, a tag powered by two 'AA' sized batteries would last for over a year and weigh approximately $40 \mathrm{~g}$ [11].

Three Spotter tags are shown in Fig. 3. These are powered by a single lithium CR123A [11] battery which provides over six months of operation. They have been encapsulated in quickset epoxy to make them robust and waterproof. The tags are fitted with a quarter wave antenna fabricated from a length of $0.5 \mathrm{~mm}$ steel wire. Proximity logs are stored on-board in the 64 kbyte FLASH memory of the microcontroller, ready for upload to Pack tags when within range.

\section{Pack tags}

Pack tags form the multi-hop network. They perform all the tasks of Spotter nodes and also route information through the wireless network in a store-and-forward fashion. As the radio radius is small compared to the total area the network is very sparse, thus information is transferred opportunistically upon contact with another Pack tag or base-station.

To decide how to route packets, nodes assess their ranking (the ranking is similar to reputation schemes in wireless security) in terms of the global distribution of resources (such as remaining battery energy or connectivity) using local information. This is dynamically assessed, and thus if new nodes (with large amounts of battery energy) are inserted into the network, these new nodes will assume a higher rank and participate more fully in the processes of routing, removing the load of routing from nearly exhausted nodes. Furthermore, as a node nears the end of its lifetime it can remove certain functionalities from the finite state machine, becoming a spotter node and ultimately a marker node. In this way, data can still be obtained from a tagged animal for much longer than if its tag was always providing a large amount of functionality. 


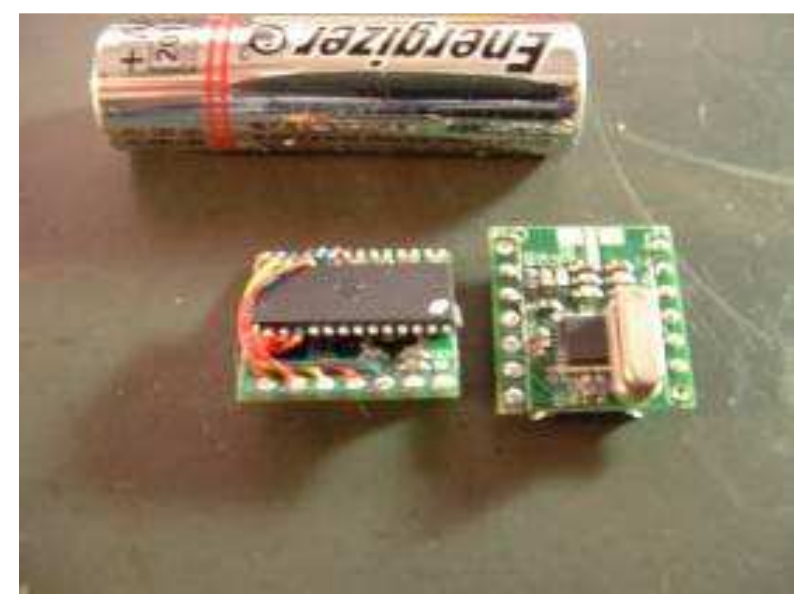

Figure 2. Two unpackaged marker tags. The microcontroller (18LF2620) can be seen on the left tag and the radio transceiver on the right tag. An 'AA' battery is shown for scale purposes.

The details of determining a node's rank within the network are beyond the scope of this paper and can be found in [7] which discusses a single copy routing strategy and [12] which extends the framework to a multi-copy routing strategy with replication and redundancy.

Pack tags transmit a special beacon packet which contains information which other tags use to update their ranking within the network as well as their ID. Thus, the beacon packet acts both as a proximity locator for the purpose of animal tracking and also as a network control or discovery packet. As the NRF905 is frequency agile, a pack tag advertises a random channel to use for network data transfer when it sends the beacon packet. This channel is separate from the beacon channel, so the act of transferring data does not affect beacon transmissions at all - in fact, the networking and the beaconing are completely distinct from one another.

Quantifying energy usage of pack tags is difficult, because it depends on a variety of factors such as the network density, volume of traffic and network composition. However, typical lifetimes are in the range of 90 days per Ah of battery capacity. Nodes thus need two alkaline ' $\mathrm{C}$ ' cells to survive for a year or more, resulting in a tag weight of approximately $150 \mathrm{~g} \mathrm{[11].} \mathrm{If}$ the tag is also equipped with a GPS receiver (which takes fixes every 15 minutes with an average fix time of 30 seconds) it requires six ' $\mathrm{C}$ ' cells, resulting in a tag weight of approximately $400 \mathrm{~g}$ [11]. This example also demonstrates the high power consumption of the GPS receiver and why it is unsuitable for use on smaller animals. This weight can be reduced significantly if the tag is equipped with a solar panel.

Fig. 4 shows a Pack tag. This is an early prototype version. It is equipped with a PIC18LF4620 microcontroller, a GPS receiver and a $512 \mathrm{Mb} \mathrm{SD}$ card for data storage. In addition to the standard Pack tag features, it also has a tri-axial accelerometer used for measuring animal motion and scheduling GPS fixes. A temperature sensor is used to monitor the ambient temperature, and a photo-sensor measures the incident light level. Our new version of this tag is greatly reduced in size and has the ability to recharge the battery using a flexible solar cell.

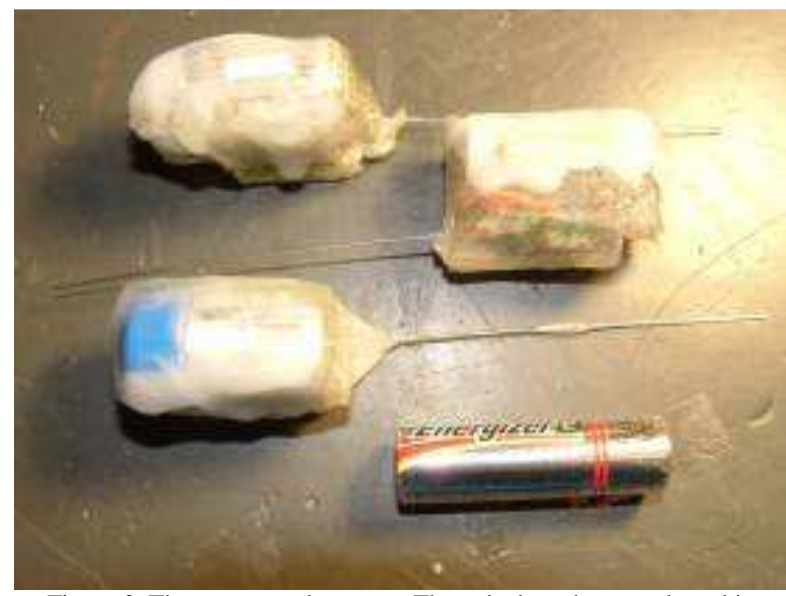

Figure 3. Three spotter class tags. The units have been packaged in epoxy resin for strength and waterproofing. The tags are equipped with a rigid quarter wave whip aerial. An 'AA' battery is shown for scale purposes.

\section{Base-station Tags}

These nodes act as sink nodes in the network and provide an interface between the wireless data gathering network and the end users. They are essentially pack tags except that all received data is forwarded out of the network via some communications interface. This interface can either be wired or wireless, depending on the application. Base-stations can be placed at convenient sites (such as at the top of a hill) or at points of attraction for animals (such as waterholes or salt licks). Base-stations can be mobile, and these can be carried by people (game-rangers or tourists), attached to vehicles or affixed to large animals, such as elephants. The choice of the communications interface depends on the local infrastructure, but can be cellular GSM modem, UHF modem or even satellite upload. As the communications interface is long range, its power consumption is typically large. Thus, base-station tags should be equipped with a solar panel or attached to a source of mains power if possible.

Base-station nodes can also be equipped with directional antennas. This will increase the accuracy of location estimates, as the bearing to a beaconing node can be determined. In addition, fixed base-stations can be equipped with more sensitive radio receivers and larger antenna, which will increase the range at which beacons can be detected. Thus, many of the well established techniques of conventional VHF tracking can be carried over to our system.

A base-station node is shown in Fig. 5. This node provides an interface between the wireless animal tracking network and a PC using a serial link. The base-station can also act in passive listening mode, where it continually listens to beacons and reports overheard beacons to the host program.

\section{RELATED WORK}

ZebraNet provided a comprehensive test on the use of wireless sensor networks for animal tracking [1], [2]. GPS equipped collars were fitted on zebras and exchange information in an epidemic fashion. Their routing algorithm is 


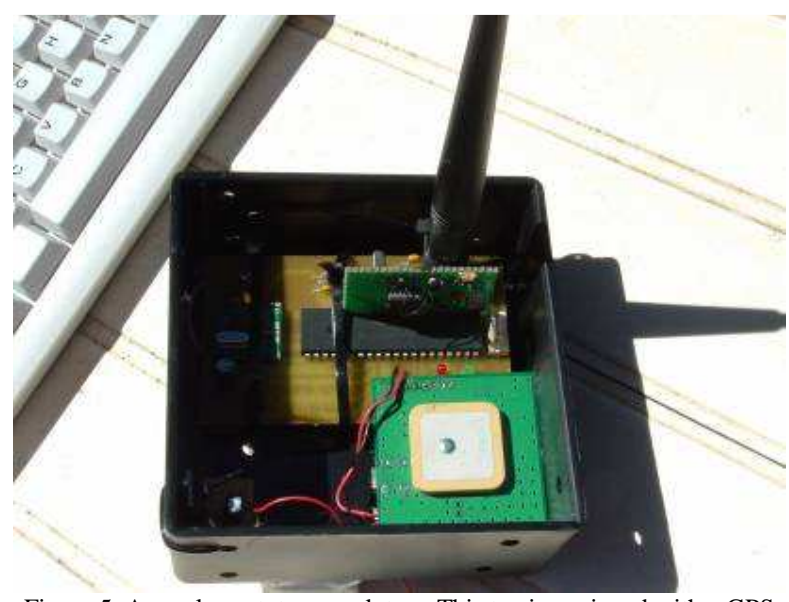

Figure 5. An early prototype pack tag. This tag is equipped with a GPS unit, tri-axial accelerometer, light and temperature sensors.

very simple and leads to buffer overflow as every node in the network stores information from every other node, thus limiting scalability. The authors only considered fitting the collars on a single type of animal. The Shared Wireless Infostation Model (SWIM) is a routing protocol that addresses some of the issues faced by the Epidemic routing protocol [13]. Their main contribution was in the form of 'anti-packets' messages that prevent nodes from buffering data that has already been delivered to the base-station. However, like ZebraNet, they concern themselves with instrumenting a single species - whales.

Sikka et al. present a wireless sensor network designed to monitor a typical farm environment, in particular cattle monitoring [14], [15]. Using the capabilities of different animals to lead to a better performing network is also not considered in their work. In addition, an agricultural deployment is much simpler as collars can be easily replaced when they fail. A similar agricultural monitoring system is discussed in [16]. Likewise, they only concern themselves with instrumenting one type of animal.

A similar scheme to ZebraNet is CenWits, which is a wireless network based Search-and-Rescue system. Nodes are equipped with GPS receivers and exchange databases of recent locations of other nodes when they meet. Based on this data, a rescue team can determine the location where a missing person was last in contact with another node [17].

Proximity detection between groups of animals has recently been undertaken using commercially available collars [18]. Like other research this only concentrated on a single animal species, namely raccoons. These collars do not form a wireless network and have to be retrieved in order to download data from them using a cable. This severely limits their usefulness for wide ranging species, and places additional stress on animals as they have to be recaptured.

To the best of our knowledge, our system is the first work which has combined the simplicity of VHF tracking and the accuracy of GPS, along with the flexibility of a wireless network communications overlay in order to track multiple species using the same basic technology.

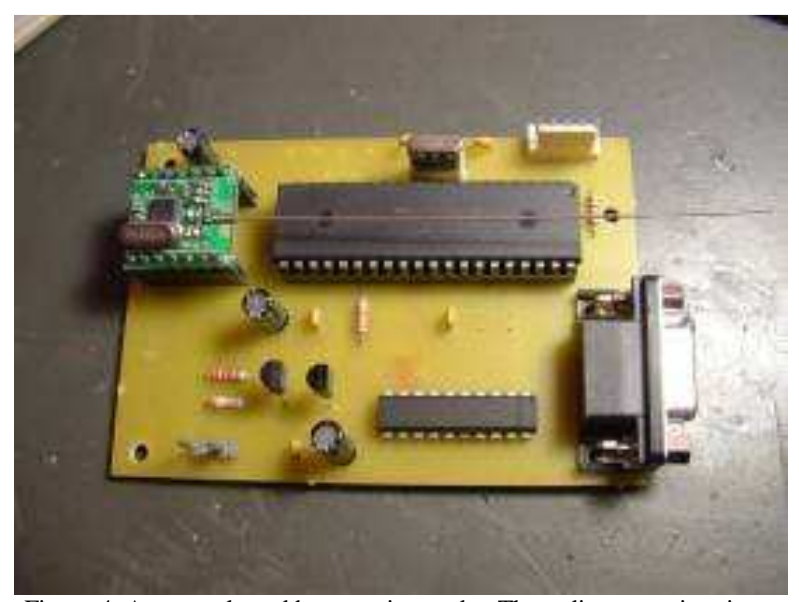

Figure 4. An unpackaged base-station node. The radio transceiver is on the left, a PIC18LF4620 microcontroller is to the right. The unit is connected to a PC via an RS-232 link.

\section{FUTURE WORK}

This work is at an advanced stage of development, with working prototype tags having been constructed. Three Spotter class tags and a base-station tag have been deployed at Ongava Research Centre in Namibia in order to test their operation in the field. The Spotter tags have been attached to vehicles, and have been operating correctly for the past three months. The next stage of the project is to deploy a small test system on wild animals. Because of the high cost of deployment (helicopter, veterinarians and tranquilizer drugs), our tags will be piggybacked onto existing commercial collars for preliminary trials. An advantage of this approach is that our retrieved data can be compared with that of the commercial collar.

The system is not only suitable for tracking animals, but can also be used to monitor a large variety of factors which influence animal behaviour. For example, water availability plays a large role in animal location. Waterholes and rivers can be equipped with water-level sensors which can relay information about the amount of water in an area through the wireless network to a researcher. Based on park-wide information on water distribution, informed decisions can be made about where to provide more sources of water. Other sensors can also be installed, such as fire detectors, soil moisture and temperature sensors amongst others. We have developed a water level sensor but have yet to test it in the field.

An important issue is field reprogramming. In order to provide firmware updates and fix errors without having to retrieve collars (which in most cases is difficult or impossible), it is desirable to be able to program the collars 'over-the-air'. We are planning to implement this feature in our next version.

\section{CONCLUSIONS}

We have presented a new system for monitoring multiple species using both GPS tracking where possible and techniques from conventional VHF tracking. Simple tags carried by small 
animals use the increased capabilities of the large tags carried on larger animals to carry data in an energy efficient manner to the end user. Information is transferred using a delay tolerant network where contacts are formed opportunistically when tags are within range of each other. By using a mixture of technologies, a wide range of different animal species can be simultaneously tracked, using the same basic hardware. This work has the potential to revolutionize the field of wildlife tracking, by unifying two currently disparate tracking technologies and meshing them with the power of a mobile wireless network.

\section{ACKNOWLEDGEMENTS}

We thank Dr Ken Stratford for his helpful comments and for deploying the tag devices in the field. Andrew Markham is supported by a UCT International Students Scholarship and thanks the Postgraduate Funding Office for their assistance.

\section{REFERENCES}

[1] P. Juang, H. Oki, Y. Wang, M. Martonosi, L. Peh and D. Rubenstein, "Energy-Efficient Computing for Wildlife Tracking: Design Tradeoffs and Early Experiences with ZebraNet", ASPLOS X, San Jose, CA, USA, 2002

[2] P. Zhang, C. M. Sadler, S. A. Lyon and M. Martonosi, "Hardware Design Experiences in ZebraNet", SenSys, Baltimore, Maryland, USA, 2004

[3] W. Cochran, "Wildlife Telemetry", In S. D. Schemnitz (Ed.), Wildlife management techniques manual, 1980

[4] R. E. Kenward, A Manual for Wildlife Radio Tagging, Academic Press, 2001

[5] Kaplan (Ed.), Understanding GPS: Principles and Applications, Artech House, 1996

[6] G. Spong, M. Johansson and M. Björklund, "High genetic variation in leopards indicates large and long-term stable effective population size", Molecular Ecology, vol. 9, no. 11, 2000 pp. 1773

[7] A. C. Markham and A. J. Wilkinson, "The Adaptive Social Hierarchy A Self Organizing Network Based on Naturally Occurring Structures", In Proceedings of the First International Conference on Biologically Inspired Networks and Computation (BIONETICS), Cavelese, Italy, 2006

[8] Microchip Technology, http://www.microchip.com

[9] Nordic Semiconductor, http://www.nordicsemi.com

[10] u-blox, http://www.u-blox.com

[11] Sanyo battery specifications, http://us.sanyo.com/batteries

[12] A. C. Markham and A. J. Wilkinson, "A biomimetic ranking system for mobile energy constrained wireless networks", In Proceedings of the Tenth Southern African Telecommunications, Networks and Applications Conference (SATNAC), Sugar Beach Resort, Mauritius, 2007

[13] T. Small, Z. J. Haas, A. Purgue and K. Fristrup "The Shared Wireless Infostation Model: A New Ad Hoc Networking Paradigm (or Where There is a Whale, There is a Way)", Fourth ACM International Symposium on Mobile AdHoc Networking \& Computing, 2003

[14] P. Sikka, P. Corke, P. Valencia, C. Crossman, D. Swain and G. BishopHurley, "Wireless adhoc sensor and actuator networks on the farm", In Proceedings of the Fifth International Conference on Information Processing in Sensor Networks, Nashville, Tennessee, USA, April 2006

[15] T. Wark, P. Corke, P. Sikka, L. Klingbeil, Y. Guo, C. Crossman, P. Valencia, D. Swain and G. Bishop-Hurley, "Transforming Agriculture through Pervasive Wireless Sensor Networks", IEEE Pervasive Computing, vol. 6, no. 2, pp. 50-57, Apr-Jun, 2007

[16] M. Radenkovic and B. Wietrzyk, "Wireless Mobile Ad-Hoc Sensor Networks for Very Large Scale Cattle Monitoring", In Proceedings of the 6th International Workshop Applications and Services in Wireless Networks (ASWN 06), 2006, pp. 47-58
[17] J.H. Huang, S. Amjad and S. Mishra, "CenWits: a sensor-based loosely coupled search and rescue system using witnesses", In Proceedings of the 3rd international conference on Embedded networked sensor systems (SenSys '05), San Diego, California, USA, 2005

[18] S. Prange, T. Jordan, C. Hunter and S D Gehrt, "New Radiocollars for the Detection of Proximity among Individuals", Wildlife Society Bulletin, vol. 34, no. 5, Dec 2006, pp. 1333 